\title{
Curriculum review: colorectal cancer surveillance and management of dysplasia in IBD
}

\author{
Hajir Ibraheim, ${ }^{1}$ Angad Singh Dhillon, ${ }^{1}$ loannis Koumoutsos, ${ }^{1}$ \\ Shraddha Gulati, ${ }^{2}$ Bu'Hussain Hayee $^{2}$
}

\begin{abstract}
'IBD Centre, Guy's and St Thomas' Hospital, London, UK ${ }^{2}$ King's Institute of Therapeutic Endoscopy, King's College London, London, UK
\end{abstract}

Correspondence to Dr Hajir Ibraheim, IBD Centre, Guy's and St Thomas' Hospital, London SE1 9RT, UK; hajir. ibraheim@gmail.com

Received 16 October 2017 Revised 20 December 2017 Accepted 14 January 2018 Published Online First 10 February 2018
D Check for updates

To cite: Ibraheim $\mathrm{H}$ Dhillon AS, Koumoutsos I, et al. Frontline Gastroenterology 2018:9:271-277.

\begin{abstract}
The significantly increased risk of colorectal cancer $(\mathrm{CRC})$ in longstanding colonic inflammatory bowel disease (IBD) justifies the need for endoscopic surveillance. Unlike sporadic CRC, IBD-related CRC does not always follow the predictable sequence of low-grade to high-grade dysplasia and finally to invasive carcinoma, probably because the genetic events shared by both diseases occur in different sequences and frequencies. Surveillance is recommended for patients who have had colonic disease for at least 8-10 years either annually, every 3 years or every 5 years with the interval dependant on the presence of additional risk factors. Currently, the recommended endoscopic strategy is high-definition chromoendoscopy with targeted biopsies, although the associated lengthier procedure time and need for experienced endoscopists has limited its uniform uptake in daily practice. There is no clear consensus on the management of dysplasia, which continues to be a challenging area particularly when endoscopically invisible. Management options include complete resection (and/or referral to a tertiary centre), close surveillance or proctocolectomy. Technical advances in endoscopic imaging such as confocal laser endomicroscopy, show exciting potential in increasing dysplasia detection rates but are still far from being routinely used in clinical practice.
\end{abstract}

\section{INTRODUCTION}

The current article offers an up to date overview of endoscopic surveillance in colonic inflammatory bowel disease (IBD) and management of associated dysplasia, while also covering the relevant competencies in the 2010 gastroenterology curriculum (figure 1).

\section{Epidemiology of IBD-related colorectal} cancer

Patients with ulcerative colitis (UC) or Crohn's colitis are at increased risk of colorectal cancer (CRC), which accounts for up to $10 \%$ of all deaths in patients with IBD. ${ }^{1}$

The seminal meta-analysis which included 54, 478 patients with UC from 116 studies, reported an overall CRC prevalence of $3.7 \%$. This corresponded to a cumulative incidence of developing CRC at 2\% by 10 years, $8 \%$ by 20 years and $18 \%$ by 30 years. $^{2}$ More recent population-based studies suggest that this risk has decreased over time and while precise reasons have not been elucidated, it is likely explained by advances in maintenance therapy and surveillance colonoscopy. ${ }^{3}$

There is less certainty regarding the incidence of CRC in Crohn's colitis but rates appear to be comparable with UC, hence, a uniform surveillance regimen is recommended for both conditions.

\section{Risk factors for IBD-related CRC}

The strongest risk factor for development of IBD-related CRC is duration of disease, with cancer rarely occurring before 7 years ${\text { of } \text { colitis. }^{2} 4}^{4}$ Other relevant risk factors include: a younger age at onset of IBD, the degree of inflammation as indicated by the extent of colonic inflammation and the presence of strictures, backwash ileitis and post-inflammatory polyps, although there is only low-quality evidence to support the latter three as being independent risk factors. ${ }^{5}$ There is compelling evidence that a family history of sporadic CRC in a first-degree relative under the age of 50, coexistence of primary sclerosing cholangitis (PSC) or the presence of a colonic stricture or dysplasia within the past 5 years, incur significant risk and justify annual colonoscopic surveillance. ${ }^{5}$ Coexistent PSC is also associated with a higher cumulative incidence of CRC or 


\section{Complications and Special Situations}

To recognise long term complications of IBD, and their treatment including medical and surgical treatment.

\section{Knowledge \\ Knows the complications of IBD including stricturing, fistulae, extraintestinal manifestation colon cancer and special situations such as pregnancy.}

Assessment $\mathrm{CbD}$, mini-CEX, SCE 1

\section{Skills}

Able to recognise potential complications and take appropriate action to investigate and alter treatment as necessary including referral for surgery and involvement of other healthcare professionals

Figure 1 The 2010 gastroenterology competencies for inflammatory bowel disease-related colorectal cancer.

dysplasia: 9\% after 10 years of colitis, $21 \%$ after 20 years and $50 \%$ after 25 years. ${ }^{6}$

\section{Pathophysiology of IBD-related CRC}

Whilst most IBD- related CRCs are adenocarcinomas, there is a higher incidence of poorly differentiated anaplastic and mucinous carcinomas compared with sporadic CRC. ${ }^{7}$

The mechanism through which chronic inflammation leads to carcinogenesis is not well defined, but it appears that similar genetic events are shared between sporadic CRC in the general population and

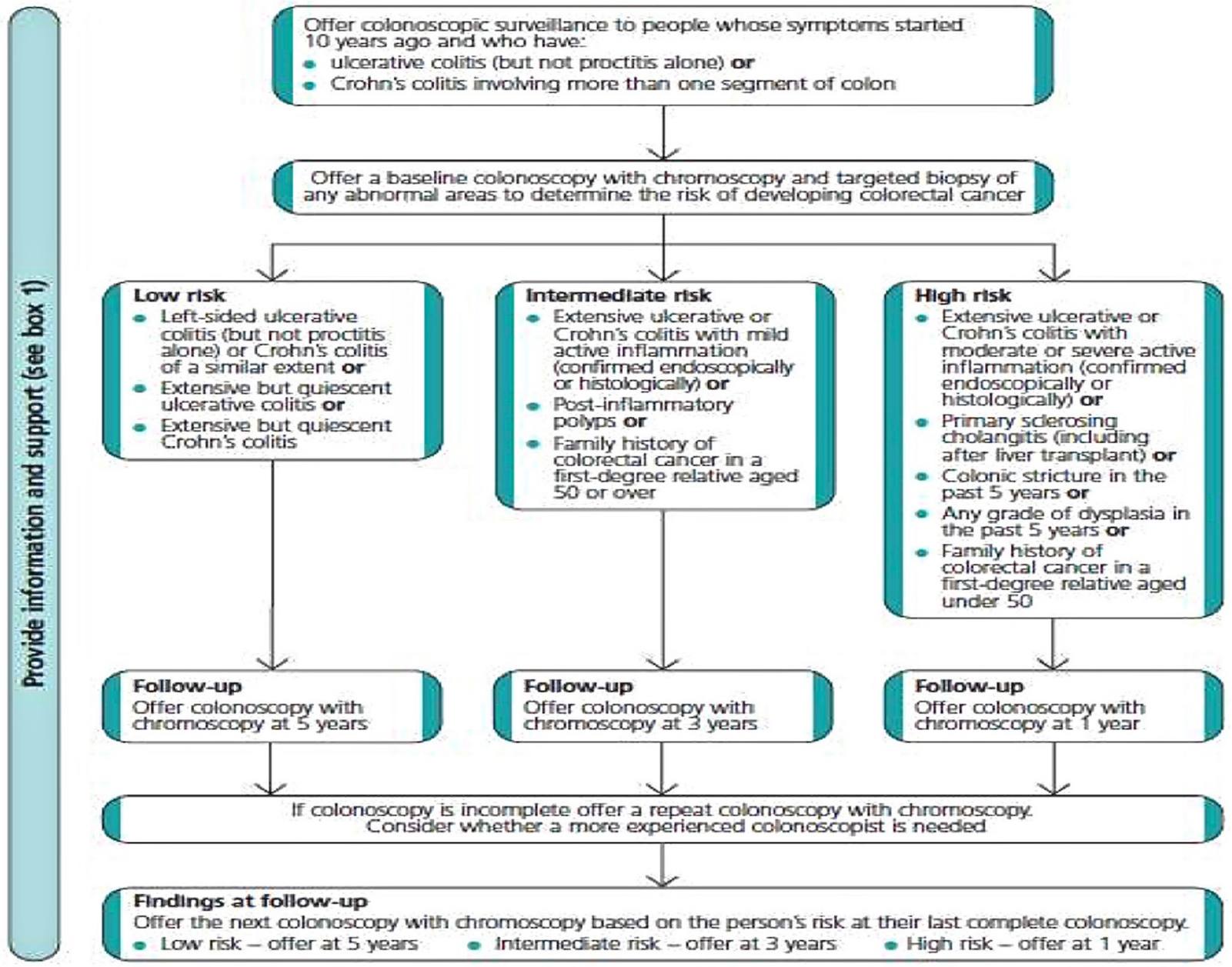

Figure 22011 National Institute for Health and Care Excellence (NICE) algorithm for determining timing of surveillance colonoscopies in the inflammatory bowel disease population. 
Box 1 The 2011 National Institute for Health and Care Excellence (NICE) guidance on risks of developing CRC in the colonic inflammatory bowel disease (IBD) population. ${ }^{5}$

\section{Low risk}

- extensive but quiescent ulcerative colitis or

- extensive but quiescent Crohn's colitis or

- left-sided ulcerative colitis (but not proctitis alone) or Crohn's colitis of a similar extent.

\section{Intermediate risk}

- extensive ulcerative or Crohn's colitis with mild active inflammation that has been confirmed endoscopically or histologically or

- postinflammatory polyps or

- family history of colorectal cancer in a first-degree relative aged 50 year or over.

\section{High risk}

- extensive ulcerative or Crohn's colitis with moderate or severe active inflammation that has been confirmed endoscopically or histologically or

- primary sclerosing cholangitis (including after liver transplant) or

- colonic stricture in the past 5 years or

- any grade of dysplasia in the past 5 years or

- family history of colorectal cancer in a first-degree relative aged under 50 years.

IBD-related CRC. These include microsatellite instability, inhibition of regulatory genes via hypermethylation of the promoter regions, loss of the tumour suppressor genes- adenomatous polyposis coli (APC) and $\mathrm{p} 53$, and activation of the oncogene K-ras. ${ }^{7}$ However, a key difference relates to the timing of these events, which occur in a different sequence and at an altered frequency. ${ }^{7}$ For example, CRC in IBD does not always follow a sequential progression from low-grade dysplasia (LGD) to high-grade dysplasia (HGD) and ultimately to carcinoma, and cancer can even occurr in patients with no prior dysplasia. ${ }^{6-8}$ One meta-analysis showed that LGD confers a nine-fold increase in risk of developing cancer, ${ }^{9}$ suggesting that the presence of dysplasia is a serious warning for the presence of carcinoma. In contrast, LGD adenomas (measuring less than $10 \mathrm{~mm}$ in size) in the non-IBD population only confers a 1.8 -fold risk of advanced neoplasia. ${ }^{10}$

Interestingly, both synchronous (simultaneous) and metachronous (consecutive) dysplasia and carcinomas occur more frequently in IBD patients than in sporadic CRC. This is probably due to the 'field effect' of cellular and molecular changes present throughout the IBD colon. ${ }^{7}$ Taken altogether, the unpredictable sequence of events in IBD-related CRC, coupled with its more aggressive nature highlights the importance of implementing robust surveillance strategies.

\section{SURVEILLANCE IN IBD}

\section{Rationale and timing for surveillance}

The aim of colonoscopic surveillance is the early detection of dysplasia, with a view to preventing or reducing mortality from CRC. The 2011 National Institute for Health and Care Excellence (NICE) guidance base the rationale for implementing surveillance predominantly on a 2006 Cochrane review which 'did not show any clear evidence that surveillance colonoscopy prolongs survival in patients with extensive colitis'. Although cancers tended to be detected at an earlier stage and these patients had a correspondingly better prognosis, lead-time bias was thought to contribute to this apparent benefit. ${ }^{11}$ Whilst NICE considered this evidence to be low quality, it was agreed that surveillance should be pursued given the unethical nature of randomising patients to a 'no surveillance' arm. Moreover, this data has to be interpreted with caution in view of more recent advances in surveillance strategies that have significantly increased dysplasia detection rates.

The 2010 British Society of Gastroenterology (BSG) and NICE guidelines recommend offering colonoscopic surveillance to patients with ulcerative pancolitis/leftsided colitis and Crohn's colitis (affecting at least one third of the colon) 10 years after onset of symptoms. The exceptions are patients with concomitant PSC, who should begin annual surveillance from the time PSC is diagnosed. ${ }^{12}$

Patients with proctitis are not thought to be at increased risk of developing CRC and should follow the standard CRC screening guidelines. Of note, while proctocolectomy abolishes the risk of CRC, it does not remove the low risk of anal cancer or cancer of the rectal cuff or ileo-anal pouch.

Further surveillance colonoscopies are suggested at intervals based on risk stratification, low risk (surveillance every 5 years), intermediate (every 3 years) and high risk (annual surveillance) (figure 2). Interestingly, there is no direct evidence guiding timing of surveillance and the recommended intervals have been determined by the Guideline Development Group (GDG) consensus. Risk factors are based on disease extent, family history of CRC (with family history of CRC age $<50$ vs age $>50$ discriminating between high risk and intermediate risk respectively), as well as the presence of previous dysplasia, post-inflammatory polyps and strictures (box 1).

\section{Surveillance strategy}

Surveillance should be performed when the patient is in clinical remission as active colitis can impair the visibility of subtle lesions and accuracy of histopathological assessment. Meticulous bowel preparation is particularly important as subtle non-polypoid (flat) lesions can be easily obscured by residual matter. Although no studies have examined the impact of inadequate bowel preparation on IBD surveillance outcomes, in the general population, it is well established that 
inadequate preparation correlates inversely with dysplasia detection and is associated with an increased burden of repeat procedures. ${ }^{13}$

There are no specific recommendations for the type of bowel preparation that should be used, but it seems sensible to emphasise adherence to the pre-procedure diet and split dose laxative regimen. Moreover, low-volume preparations may be considered in patients with known stenosing or stricturing complications, previous bowel surgery or previous preparation intolerance to promote adherence.

In terms of surveillance technique, traditional white light endoscopy (WLE) is still used with 2-4 random biopsies taken every $10 \mathrm{~cm}$ in order to enhance chances of detecting flat endoscopically invisible lesions. ${ }^{5}$ Although random biopsies sample less than $0.1 \%$ of the surface mucosa, they account for dysplasia detection in up to $2.6 \%$ of patients with IBD. ${ }^{14}$ However, this technique is laborious, expensive and overall has a low diagnostic yield, prompting a shift to surveillance strategies that obviate the need for random biopsies.

Both international (Surveillance for Colorectal Endoscopic Neoplasia Detection and Management in Inflammatory Bowel Disease Patients: International Consensus (SCENIC), The European Crohn's and Colitis Organization (ECCO)) and national (NICE and BSG) guidelines strongly recommend utilising chromoendoscopy (CE) with targeted biopsies as the standard surveillance practice. CE involves spraying either a non-absorptive blue contrast agent; indigo-carmine or an absorptive stain; methylene blue in accordance with the SURFACE guidelines, using a disposable spray catheter to achieve an even application. CE enhances visualisation of subtle mucosal changes suggestive of dysplasia that might otherwise not be visible with white light (figure 3). There is convincing high-quality evidence deomonstrating that targeted biopsies with $\mathrm{CE}$ increases the yield of dysplasia detection up to 4.5 -fold. ${ }^{51415}$ Colitis-associated dysplasia is often flat/ non-polypoid with subtle changes easily missed with standard WLE. Furthermore, discriminating between dysplastic and inflammatory changes poses an additional challenge. In practice, for safety, all 'abnormal' lesions should be biopsied unless a definite visual diagnosis can be made.

Although there is no clear consensus on the role of random biopsies in CE, the BSG do not endorse random sampling while SCENIC experts are 'on the fence'. A pooled analysis showed that at most, 1.5\% of patients with dysplasia may be missed by not performing random biopsies in CE. ${ }^{14}$

Despite highly encouraging data, uptake of CE has been variable in part because it requires more time (11 min longer than WLE). necessitating three-unit endoscopy slots instead of two as per BSG recommendations. ${ }^{12}$ Moreover, CE requires additional consumables and an experienced endoscopist who is familiar with identifying suspicious mucosal patterns.
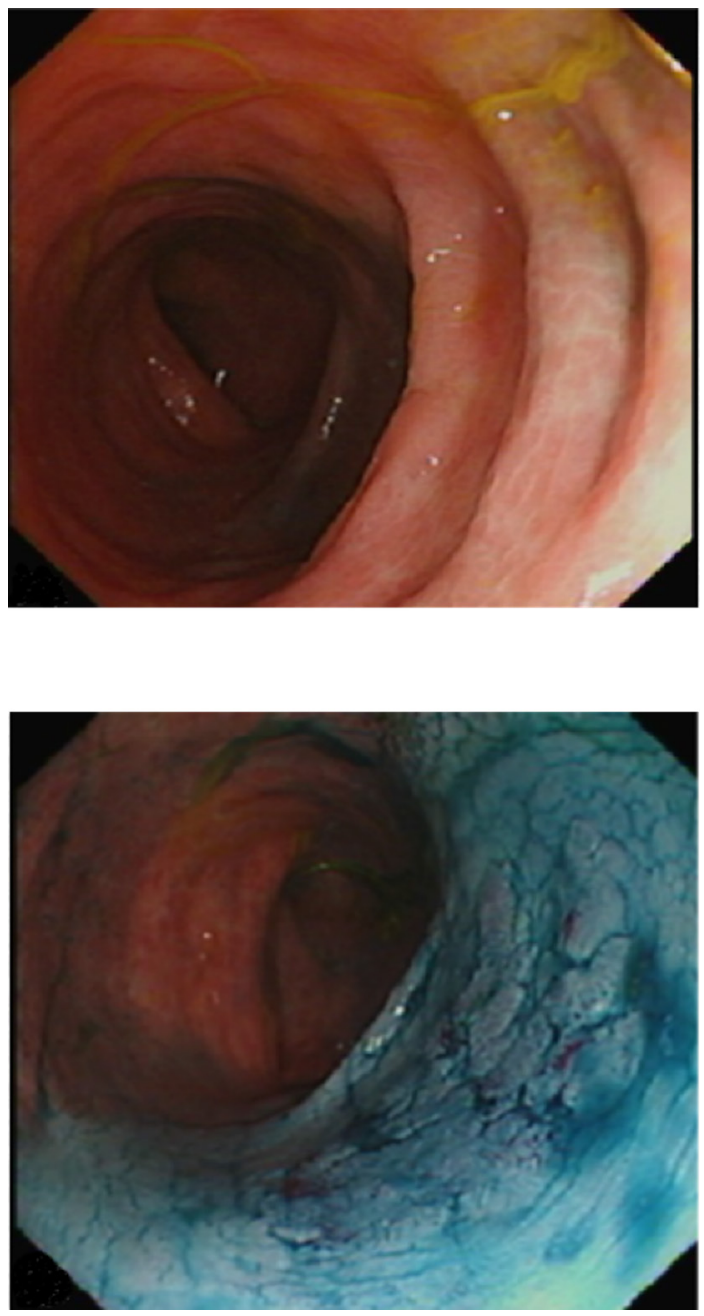

Figure 3 Endoscopic images show the same segment of colon with. Top: white light (Olympus CF-H260AZL colonoscope). Bottom: after chromoendoscopy with $0.2 \%$ indigo carmine, revealing a discrete, flat lesion (Paris IIb, LST-G) with benign pit pattern, suitable for endoscopic resection.

Although the prospect of using narrow-band imaging (NBI) as a surveillane strategy is appealing, there is no evidence that NBI increases dysplasia detection in IBD. Whilst it cannot be used as a substitute for CE or WLE surveillance, NBI can be used as an adjunct. ${ }^{12} 14$

Endoscopic advancements have led to the emergence of confocal laser endomicroscopy (CLE) which allows the endoscopist to obtain real-time histology-level images of the gastrointestinal (GI) mucosa. Once a suspicious area of mucosa is identified, contrast agents are administered and an examination of the targeted area is performed by placing the tip of the endoscope against the mucosa. High-resolution images are captured and digitally stored. Although there is promising data suggesting enhanced dysplasia detection rates, the clinical application of CLE is hampered by the need to have GI pathologists on site, a steep learning curve for the endoscopist and the overall high costs incurred. 
Table 1 The SCENIC consensus for the nomenclature of dysplasia in inflammatory bowel disease using the Paris classification, with modifications to describe ulceration and the borders of the lesion ${ }^{14}$

\begin{tabular}{|c|c|}
\hline Term & Definition \\
\hline Visible dysplasia & $\begin{array}{l}\text { Dysplasia identified on targeted biopsies from a } \\
\text { lesion visualised at colonoscopy }\end{array}$ \\
\hline Polypoid & $\begin{array}{l}\text { Lesion protruding from the mucosa into the } \\
\text { lumen } \geq 2.5 \mathrm{~mm}\end{array}$ \\
\hline Pedunculated & Lesion attached to the mucosa by a stalk \\
\hline Sessile & $\begin{array}{l}\text { Lesion not attached to the mucosa by a stalk: } \\
\text { entire base is contiguous with the mucosa }\end{array}$ \\
\hline Non-polypoid & $\begin{array}{l}\text { Lesion with little }(<2.5 \mathrm{~mm}) \text { or no protrusion } \\
\text { above the mucosa }\end{array}$ \\
\hline Superficial elevated & $\begin{array}{l}\text { Lesion with protrusion but }<2.5 \mathrm{~mm} \text { above the } \\
\text { lumen ( }<\text { height of the closed cup of a biopsy } \\
\text { forceps) }\end{array}$ \\
\hline Flat & Lesion without protrusion above the mucosa \\
\hline Depressed & $\begin{array}{l}\text { Lesion with at least a portion depressed below } \\
\text { the level of the mucosa }\end{array}$ \\
\hline \multicolumn{2}{|l|}{ General descriptors } \\
\hline Ulcerated & $\begin{array}{l}\text { Ulceration (fibrinous-appearing base with } \\
\text { depth) within the lesion }\end{array}$ \\
\hline \multicolumn{2}{|l|}{ Border } \\
\hline Distinct border & $\begin{array}{l}\text { Lesion's border is discrete and can be } \\
\text { distinguished from surrounding mucosa }\end{array}$ \\
\hline Indistinct border & $\begin{array}{l}\text { Lesion's border is not discrete and cannot be } \\
\text { distinguished from surrounding mucosa }\end{array}$ \\
\hline Invisible dysplasia & $\begin{array}{l}\text { Dysplasia identified on random (non-targeted) } \\
\text { biopsies of colon mucosa without a visible } \\
\text { lesion }\end{array}$ \\
\hline
\end{tabular}

\section{DYSPLASTIC LESIONS IN IBD}

Abnormal biopsies should be reviewed by an expert GI pathologist as dysplasia can be difficult to distinguish from epithelial regeneration secondary to inflammation. ${ }^{7}$ Because of unfavourable rates of interobserver variability, it is recommended that two GI pathologists agree on a diagnosis of dysplasia. ${ }^{12}$

Dysplasia associated with IBD is now categorised as 'endoscopically visible' and 'invisible' and shall be discussed as such here. This encompasses the original Paris classification, with the addition of modifications to describe the borders of lesions and the presence of ulceration (table 1).

It is unclear if the risk of CRC in IBD is the same for polypoid and non-polypoid dysplastic lesions, hence, both are assumed to have similar risk although studies in patients without IBD suggest that the molecular biology of non-polypoid CRC lesions differ from polypoid CRC lesions.

\section{Management of dysplastic lesions}

Management of IBD associated dysplasia remains fraught with challenges and in every instance, a multidisciplinary approach is advocated for all cases.
Endoscopically visible dysplasia

Endoscopically visible dysplasia can be categorised into polypoid and non-polypoid lesions. A detailed assessment is imperative to determine feasibility of endoscopic resection. Features favouring endoscopic resection include the presence of circumferential distinct margins, the absence of ulceration and stable access to the lesion. Advanced imaging techniques, such as magnification CE for pit pattern analysis and NBI for vascular pattern analysis may not only distinguish dysplastic from inflammatory lesions but also allow assessment of grade of dysplasia and estimate depth of submucosal invasion. ${ }^{1617}$ Referral to a tertiary endoscopic centre is encouraged, particularly for large $(>2 \mathrm{~cm})$ and/or non-pedunculated lesions which can be technically challenging to resect by conventional endoscopic methods. Non-polypoid morphology and the presence of submucosal fibrosis, not infrequently encountered in colitic colons, increase the risk of incomplete resection or adverse events. ${ }^{18}$ A range of endoscopic resection methods have been implemented successfully, including endoscopic mucosal resection, endoscopic submucosal dissection and hybrid techniques.

There is no unifying approach on the optimal surveillance strategy following successful endoscopic resection, but it is widely accepted that this should be close-initially 3-6 months' post-resection to assess for early recurrence of the lesion and annually thereafter.

SCENIC and BSG guidelines recommend biopsying mucosa adjacent to the resection area as a marker of assurance of complete endoscopic resection ${ }^{14}$. However, recent data suggests this has a low yield for dysplasia detection especially in the presence of CE. ${ }^{19}$

Endoscopically invisible dysplasia

Low-grade dysplasia

For LGD in endoscopically invisible lesions, referral to an experienced IBD surveillance endoscopist is encouraged. Further management, if dysplasia is still deemed endoscopically invisible, should be discussed in a multidisciplinary team setting taking into account disease activity, additional risk factors for CRC and patient preference for close endoscopic surveillance (6 monthly-yearly) versus colectomy. Although there have been no studies comparing surveillance colonoscopy and colectomy for endoscopically invisible dysplasia, pooled data from four studies showed that over a mean follow-up of $15-50$ months, CRC developed in seven out of 122 patients $(6 \%$, range $3 \%-9 \%)^{14}$

\section{High-grade dysplasia}

Traditionally, colectomy was advocated for the management of invisible HGD, based on historic data suggesting high rates of synchronous or metachronous cancer. ${ }^{20}$ However, most of this data is from the pre-CE and high-definition WLE era where since 
its implementation, invisible dysplasia accounts for a much lower proportion of dysplasia (less than 10\% of total dysplasia). ${ }^{14}$

The management of invisible HGD is similar to its LGD counterpart. Repeat examination by an expert endoscopist with the use of high-definition $\mathrm{CE}$ is recommended to determine whether the lesion is endoscopically visible and therefore resectable. In such a situation, the lesion should be completely resected and the patient may remain in an intense surveillance programme. If still deemed non-resectable, the next step involves obtaining repeat biopsies which are reviewed by two expert GI pathologists to confirm HGD, and further management discussed in a multidisciplinary environment. The relative risks of continued intense surveillance vs colectomy should be discussed with the patient. ${ }^{14}$

\section{CONCLUSION}

The risk of CRC in Crohn's colitis and UC increases after 8-10years of disease. While sporadic CRC and IBD-related CRC share similar genetic mutations, critical differences in the sequence and frequency of genetic events may account for why IBD-related CRC's are more obscure and potentially aggressive. The duration of colonic inflammation confers the strongest influence on CRC risk.

Currently, the surveillance technique of choice is CE with targeted biopsies, representing a paradigm shift from the low yield, laborious random biopsy technique. Dysplastic lesions are described as endoscopically invisible or endoscopically visible (polypoid and non-polypoid). Referral to tertiary endoscopic centres for further assessment and management is recommended for large lesions, non-polypoid and endoscopically invisible dysplasia.

\section{SCE QUESTIONS}

1. A 39-year-old patient with UC has had her disease for 10 years and qualifies for surveillance endoscopy. She has had multiple courses of steroids and is on azathioprine and a 5-ASA.

Which of the following factors, if present, would suggest the need for three yearly surveillance?
A. Her father having a diagnosis of CRC at 48 years.
B. Severe proctitis
C. Presence of LGD, which was completely resected on a previous colonoscopy 4 years ago
D. Post-inflammatory polyps
E. Extensive UC with moderate active inflammation confirmed histologically on the last endoscopy

\section{Answer: D}

According to the NICE and BSG guidance, annual surveillance is justified in patients with a first-degree relative diagnosed with CRC aged $<50$ years (A), any grade of dysplasia diagnosed in the last 5 years (C) and extensive UC with moderate active inflammation confirmed histologically or endoscopically (E). Other factors include the presence of concomitant PSC or colonic strictures in the past 5 years.

Proctitis is not an indication for surveillance (B) regardless of the severity.

The presence of post-inflammatory polyps is an indication for three yearly surveillance (D).

2. A 58-year-old patient with extensive Crohn's colitis is found to have a $1.5 \mathrm{~cm}$ polypoid lesion with LGD.

From the following course of actions, which is not indicated in the immediate management of this case?
A. Discussion in a multidisciplinary team (MDT) setting
B. Complete resection (en bloc)
C. Consideration for colectomy
D. Referral to a specialist IBD centre
E. Repeat surveillance endoscopy in 6 months.

\section{Answer: $\mathrm{E}$}

Dysplasia which is potentially resectable should always be managed with complete resection regardless of the grade. Close surveillance is an option for when lesions are endoscopically invisible and not easily resectable, but patients should also be given the option of a colectomy.

Referral to an MDT or specialist IBD centre are also reasonable options. Consideration of colectomy (C) is not the first choice, but if the patient had a family history of CRC or previous dysplasia, or is the patient's preference, it is potentially an option.

Contributors HI: manuscript planning, writing, editing; submission, responsible for overall content. ASD, IK and SG: manuscript planning and editing. $\mathrm{BH}$ : manuscript planning, editing, provision of original images, responsible for overall content.

Funding This research received no specific grant from any funding agency in the public, commercial or not-for-profit sectors.

Competing interests None declared.

Provenance and peer review Not commissioned; externally peer reviewed.

(C) Article author(s) (or their employer(s) unless otherwise stated in the text of the article) 2018. All rights reserved. No commercial use is permitted unless otherwise expressly granted.

\section{REFERENCES}

1 Munkholm P. Review article: the incidence and prevalence of colorectal cancer in inflammatory bowel disease. Aliment Pharmacol Ther 2003;18(Suppl 2):1-5.

2 Eaden JA, Abrams KR, Mayberry JF. The risk of colorectal cancer in ulcerative colitis: a meta-analysis. Gut 2001;48:526-35.

3 Castaño-Milla C, Chaparro M, Gisbert JP. Systematic review with meta-analysis: the declining risk of colorectal cancer in ulcerative colitis. Aliment Pharmacol Ther 2014;39:645-59.

4 Triantafillidis JK, Nasioulas G, Kosmidis PA. Colorectal cancer and inflammatory bowel disease: epidemiology, risk factors, mechanisms of carcinogenesis and prevention strategies. Anticancer Res 2009;29:2727-37.

5 NICE CfCPa. Colonoscopic surveillance for prevention of colorectal cancer in people with ulcerative colitis, Crohn's disease or adenomas, 2011. 
6 Barral M, Dohan A, Allez M, et al. Gastrointestinal cancers in inflammatory bowel disease: an update with emphasis on imaging findings. Crit Rev Oncol Hematol 2016;97:30-46.

7 Mattar MC, Lough D, Pishvaian MJ, et al. Current management of inflammatory bowel disease and colorectal cancer. Gastrointest Cancer Res 2011;4:53.

8 Ahmadi A, Polyak S, Draganov PV. Colorectal cancer surveillance in inflammatory bowel disease: the search continues. World J Gastroenterol 2009;15:61.

9 Thomas T, Abrams KA, Robinson RJ, et al. Meta-analysis: cancer risk of low-grade dysplasia in chronic ulcerative colitis. Aliment Pharmacol Ther 2007;25:657-68.

10 Hassan C, Gimeno-García A, Kalager M, et al. Systematic review with meta-analysis: the incidence of advanced neoplasia after polypectomy in patients with and without low-risk adenomas. Aliment Pharmacol Ther 2014;39:905-12.

11 Collins PD, Mpofu C, Watson AJ, et al. Strategies for detecting colon cancer and/or dysplasia in patients with inflammatory bowel disease. Cochrane Database Syst Rev 2006:CD000279.

12 Cairns SR, Scholefield JH, Steele RJ, et al. Guidelines for colorectal cancer screening and surveillance in moderate and high risk groups (update from 2002). Gut 2010;59:666-89.

13 Clark BT, Rustagi T, Laine L. What level of bowel prep quality requires early repeat colonoscopy: systematic review and meta-analysis of the impact of preparation quality on adenoma detection rate. Am J Gastroenterol 2014;109:1714-23.
14 Laine L, Kaltenbach T, Barkun A, et al. SCENIC international consensus statement on surveillance and management of dysplasia in inflammatory bowel disease. Gastrointest Endosc 2015;81:489-501.

15 Hurlstone DP, Sanders DS, Lobo AJ, et al. Indigo carmineassisted high-magnification chromoscopic colonoscopy for the detection and characterisation of intraepithelial neoplasia in ulcerative colitis: a prospective evaluation. Endoscopy 2005;37:1186-92.

16 Wada Y, Kudo SE, Misawa M, et al. Vascular pattern classification of colorectal lesions with narrow band imaging magnifying endoscopy. Dig Endosc 2011;23(Suppl 1):10611.

17 Matsuda T, Fujii T, Saito Y, et al. Efficacy of the invasive/noninvasive pattern by magnifying chromoendoscopy to estimate the depth of invasion of early colorectal neoplasms. Am J Gastroenterol 2008;103:2700-6.

18 Rutter MD, Chattree A, Barbour JA, et al. British Society of Gastroenterology/Association of Coloproctologists of Great Britain and Ireland guidelines for the management of large non-pedunculated colorectal polyps. Gut 2015;64:1847-73.

19 Ten Hove JR, Mooiweer E, Dekker E, et al. Low rate of dysplasia detection in mucosa surrounding dysplastic lesions in patients undergoing surveillance for inflammatory bowel diseases. Clin Gastroenterol Hepatol 2017;15:222-8.

20 Rutter MD, Saunders BP, Wilkinson KH, et al. Thirty-year analysis of a colonoscopic surveillance program for neoplasia in ulcerative colitis. Gastroenterology 2006;130:1030-8. 